\title{
Self-Adjoint Extension and Spectral Theory of a Linear Relation in a Hilbert Space
}

\author{
Keshav Raj Acharya \\ Department of Mathematics, Southern Polytechnic State University, Marietta, GA 30060, USA \\ Correspondence should be addressed to Keshav Raj Acharya; kacharya@spsu.edu
}

Received 21 December 2013; Accepted 28 January 2014; Published 5 March 2014

Academic Editors: B. Franchi and G. L. Karakostas

Copyright (C) 2014 Keshav Raj Acharya. This is an open access article distributed under the Creative Commons Attribution License, which permits unrestricted use, distribution, and reproduction in any medium, provided the original work is properly cited.

The aim of this paper is to develop the conditions for a symmetric relation in a Hilbert space $\mathscr{H}$ to have self-adjoint extensions in terms of defect indices and discuss some spectral theory of such linear relation.

\section{Introduction}

In this paper, we discuss the theory of linear relations in a Hilbert space. These linear relations were first studied by Arnes, Coddington, Dijksma, de Snoo, and Hassi et al in [1-4]. It has also been studied extensively more recently in [5]. The theory has particular interest because, in some of the application problems, a linear operator can have multivalued part; for example, see [6,7]. Here, we concentrate on establishing the conditions for symmetric relations to have self-adjoint extensions in terms of defect indices. Moreover, we discuss the spectral theory of such self-adjoint relations. The analogous treatment on operator theory of some of the theorems on this paper can be found in [8].

Let $\mathscr{H}$ be a Hilbert space over $\mathbb{C}$ and denote by $\mathscr{H}^{2}$ the Hilbert space $\mathscr{H} \oplus \mathscr{H}$. A linear relation $\mathscr{R}=\{(f, g): f, g \in$ $\mathscr{H}\}$ on $\mathscr{H}$ is a subspace of $\mathscr{H}^{2}$. The graph of an operator is an example of a linear relation but note that a relation can have multivalued part.

These relations have been used in some of the eigenvalue problems in ordinary differential equations. For example, the canonical systems

$$
J u^{\prime}(x)=z H(x) u(x), \quad z \in \mathbb{C},
$$

where $J=\left(\begin{array}{cc}0 & -1 \\ 1 & 0\end{array}\right)$ and $H(x)$ is a $2 \times 2$ positive semidefinite matrix whose entries are locally integrable, induce a multivalued linear relation. For instance, we may think of writing the systems in the form

$$
H(x)^{-1} J u^{\prime}=z u
$$

and consider it as an operator on a Hilbert space. But $H(x)$ is not invertible in general therefore can not be considered as an eigenvalue equation of an operator. Instead, the system induces a linear relation that may have a multivalued part. This is one of the main motivations for our work in this paper. The boundary value problem of such canonical systems has been studied by using linear relations; see $[6,7,9]$.

$D(\mathscr{R})=\{f \in \mathscr{H}:(f, g) \in \mathscr{R}\}$ and $R(\mathscr{R})=\{g \in \mathscr{H}:$ $(f, g) \in \mathscr{R}\}$ are respectively defined as the domain and range of the relation $\mathscr{R}$.

$\mathscr{R}^{-1}=\{(g, f):(f, g) \in \mathscr{R}\}$ denotes the inverse relation. The adjoint of $\mathscr{R}$ on $\mathscr{H}$ is a closed linear relation defined by

$$
\mathscr{R}^{*}=\left\{(h, k) \in \mathscr{H}^{2}:\langle g, h\rangle=\langle f, k\rangle, \forall(f, g) \in \mathscr{R}\right\} .
$$

A linear relation $\mathcal{S}$ is called symmetric if $\mathcal{S} \subset \mathcal{S}^{*}$ and selfadjoint if $\mathcal{S}=\mathcal{S}^{*}$. From now on, we write relation to mean linear relation.

A relation $\mathscr{R}$ is called isometry if

$$
\left\langle f_{1}, f_{2}\right\rangle=\left\langle g_{1}, g_{2}\right\rangle, \quad \forall\left(f_{1}, g_{1}\right),\left(f_{2}, g_{2}\right) \in \mathscr{R}
$$

and $\mathscr{R}$ is unitary if it is isometry and $D(\mathscr{R})=R(\mathscr{R})=\mathscr{H}$. Let $(z-\mathscr{R})=\{(f, z f-g):(f, g) \in \mathscr{R}\}, N(\mathscr{R}, z)=\{f:$ $(f, z f) \in \mathscr{R}\}$ and $\mathscr{R}_{z}=R(z-\mathscr{R})$.

It is clear to see that $N\left(\mathscr{R}^{*}, \bar{z}\right)=\mathscr{R}_{z}^{\perp}$.

In Section 2, we establish the condition for a symmetric relation to have self-adjoint extensions in terms of defect indices and in Section 3 we discuss spectral theory. 


\section{Defect Indices and Self-Adjoint Extension}

Let $\mathscr{R}$ be a relation on a Hilbert space $\mathscr{H}$. The set

$$
\begin{gathered}
\Gamma(\mathscr{R})=\{z \in \mathbb{C}: \text { there exists a } C(z)>0 \text { such that } \\
\|(z f-g)\| \geq C(z)\|f\|, \forall(f, g) \in \mathscr{R}\}
\end{gathered}
$$

is defined as the regularity domain of $\mathscr{R}$ and $S(\mathscr{R})=\mathbb{C}-\Gamma(\mathscr{R})$ is defined as the Spectral Kernel of $\mathscr{R}$. Note that

(i) $z \in \Gamma(\mathscr{R})$ if and only if $(z-\mathscr{R})^{-1}$ is a bounded linear operator on $\mathscr{H}$;

(ii) if $\mathscr{R}$ is symmetric, then $\mathbb{C}-\mathbb{R} \subset \Gamma(\mathscr{R})$;

(iii) $\Gamma(\mathscr{R})$ is open.

The subspace $\mathscr{R}_{z}^{\perp}$ is called the defect space of $\mathscr{R}$ and $z$. The cardinal number $\beta(\mathscr{R}, z)=\operatorname{dim} \mathscr{R}_{z}^{\perp}$ is called the defect index of $\mathscr{R}$ and $z$.

Theorem 1. The defect index $\beta(\mathscr{R}, z)$ is constant on each connected subset of $\Gamma(\mathscr{R})$. If $\mathscr{R}$ is symmetric, then the defect index is constant on the upper and lower half-planes.

Proof. Let $Q_{z}$ denote the orthogonal projection onto $\overline{\mathscr{R}}_{z}$. We first show that $\left\|Q_{z}-Q_{z_{0}}\right\| \rightarrow 0$ as $z \rightarrow z_{0}$, for any $z_{0} \in \Gamma(\mathscr{R})$. Let $z_{0} \in \Gamma(\mathscr{R})$; then there is a constant $C\left(z_{0}\right)>0$ such that

$$
\|f\| \leq C\left(z_{0}\right)\left\|z_{0} f-g\right\|,
$$

for all $(f, g) \in \mathscr{R}$. For $\left|z-z_{0}\right|<1 /\left(2 C\left(z_{0}\right)\right)$ and all $(f, g) \in \mathscr{R}$, we have

$$
\begin{gathered}
\|f\| \leq C\left(z_{0}\right)\left\|z_{0} f-g\right\| \leq\left(\|z f-g\|+\left|z-z_{0}\right|\|f\|\right) \\
\leq C\left(z_{0}\right)\|z f-g\|+\frac{1}{2}\|f\| \\
\therefore\|f\| \leq C\left(z_{0}\right)\|z f-g\| .
\end{gathered}
$$

For $h \in R\left(Q_{z_{0}}\right)^{\perp}=\mathscr{R}_{z_{0}}^{\perp}$,

\section{$\left\|Q_{z} h\right\|$}

$$
\begin{gathered}
=\sup \left\{|\langle h, z f-g\rangle|: z f-g \in \mathscr{R}_{z},\|z f-g\| \leq 1\right\} \\
=\sup \left\{\left|\left\langle h,\left(z-z_{0}\right) f\right\rangle\right|: z f-g \in \mathscr{R}_{z},\|z f-g\| \leq 1\right\} . \\
\therefore\left\|Q_{z} h\right\| \leq\|h\|\left|z-z_{0}\right| C\left(z_{0}\right) .
\end{gathered}
$$

Similarly, for $h \in R\left(Q_{z}\right)^{\perp}=\mathscr{R}_{z}^{\perp}$,

$$
\left\|Q_{z_{0}} h\right\| \leq\|h\|\left|z-z_{0}\right| C\left(z_{0}\right) .
$$

It follows that

$$
\left\|Q_{z}-Q_{z_{0}}\right\| \leq 2\left|z-z_{0}\right| C\left(z_{0}\right) .
$$

Let $P_{z}$ denote an orthogonal projection onto $\overline{\mathscr{R}}_{z}^{\perp}$; then

$$
\begin{array}{r}
\left\|p_{z}-p_{z_{0}}\right\|=\left\|Q_{z}-Q_{z_{0}}\right\| \leq 2\left|z-z_{0}\right| C\left(z_{0}\right) \longrightarrow 0 \\
\text { as } z \longrightarrow z_{0} .
\end{array}
$$

Hence, if we choose $0<\epsilon<1 /\left(2 C\left(z_{0}\right)\right)$, then $\left\|p_{z}-p_{z_{0}}\right\|<1$, for $\left|z-z_{0}\right|<\epsilon$. Therefore, $\operatorname{dim} \mathscr{R}_{z}^{\perp}=\operatorname{dim} \mathscr{R}_{z_{0}}^{\perp}$. It follows that

$$
\beta(\mathscr{R}, z)=\beta\left(\mathscr{R}, z_{0}\right) \text { for }\left|z-z_{0}\right|<\epsilon .
$$

If $\mathscr{R}$ is symmetric, then the upper and lower half-planes are connected subsets of $\Gamma(\mathscr{R})$; therefore, the defect index is constant there.

Letting $\mathscr{R}$ be a symmetric relation on a Hilbert space, for $z \in \mathbb{C}^{+}$, the defect index $m=\beta(\mathscr{R}, z)$ and, for $w \in \mathbb{C}^{-}$, the defect index $n=\beta(\mathscr{R}, w)$ are written as a pair $(m, n)$ and are called the defect indices of $\mathscr{R}$.

The Cayley transform of a symmetric relation $\mathscr{R}$ on $\mathscr{H}$ is defined by the relation

$$
\mathscr{V}=\{(g+i f, g-i f):(f, g) \in \mathscr{R}\} .
$$

Then clearly $D(\mathscr{V})=R(\mathscr{R}+i)$ and $R(\mathscr{V})=R(\mathscr{R}-i)$.

Theorem 2. If $\mathscr{R}$ is a symmetric relation on $\mathscr{H}$ and $\mathscr{V}$ is the Cayley transform of $\mathscr{R}$, then,

(1) $\mathscr{V}$ is isometry;

(2) $R(I-\mathscr{V})=D(\mathscr{R})$ and $\mathscr{R}=\{(f-g, i(f+g)):(f, g) \in$ $\mathscr{V}\}$

(3) $\mathscr{R}$ is multi-valued if and only if $N(I-\mathscr{V}) \neq\{0\}$.

Proof. (1) Let $\left(u_{1}, v_{1}\right),\left(u_{2}, v_{2}\right) \in \mathscr{V}$; then $u_{i}=g_{i}+i f_{i}$ and $v_{i}=g_{i}-i f_{i}$, for $\left(f_{i}, g_{i}\right) \in \mathscr{R}, i=1,2$; then

$$
\begin{aligned}
\left\langle u_{1}, u_{2}\right\rangle & =\left\langle g_{1}+i f_{1}, g_{2}+i f_{2}\right\rangle \\
& =\left\langle g_{1}, g_{2}\right\rangle+\left\langle g_{1}, i f_{2}\right\rangle+\left\langle i f_{1}, g_{2}\right\rangle+\left\langle i f_{1}, i f_{2}\right\rangle \\
& =\left\langle g_{1}, g_{2}\right\rangle+i\left\langle g_{1}, f_{2}\right\rangle-i\left\langle f_{1}, g_{2}\right\rangle-i^{2}\left\langle f_{1}, f_{2}\right\rangle \\
& =\left\langle g_{1}-i f_{1}, g_{2}-i f_{2}\right\rangle \\
& =\left\langle v_{1}, v_{2}\right\rangle .
\end{aligned}
$$

(2) It follows from the definition of Cayley transform.

(3) Suppose that $\mathscr{R}$ is multivalued; then there is $g \in$ $\mathscr{H}, g \neq 0$ such that $(0, g) \in \mathscr{R}$. It follows by definition of $\mathscr{V}$ that $(g, g) \in \mathscr{V}$. Hence, $g \in N(I-\mathscr{V})$. On the other hand, let $g \in N(I-\mathscr{V}), g \neq 0$; then $(g, g) \in \mathscr{V}$; then, by (2), $(0,2 i g) \in \mathscr{R}$. Hence, $\mathscr{R}$ is multivalued.

Theorem 3. A relation $\mathscr{V}$ on $\mathscr{H}$ is the Cayley transform of a symmetric relation $\mathscr{R}$ if and only if $\mathscr{V}$ has the following properties.

(1) $\mathscr{V}$ is an isometric relation.

(2) $R(I-\mathscr{V})=D(\mathscr{R})$.

The relation $\mathscr{R}$ is given by $\mathscr{R}=\{(f-g, i(f+g)):(f, g) \in \mathscr{V}\}$.

$\mathscr{V}$ satisfies the properties (1) and (2). Conversely, supposing 
that $\mathscr{V}$ has properties (1) and (2), we show that $\mathscr{R}=\{(f-$ $g, i(f+g)):(f, g) \in \mathscr{V}\}$ is a symmetric relation. then

Suppose $\left(f_{1}-g_{1}, i\left(f_{1}+g_{1}\right)\right),\left(f_{2}-g_{2}, i\left(f_{2}+g_{2}\right)\right) \in \mathscr{R}$;

$$
\begin{aligned}
& \left\langle i\left(f_{1}+g_{1}\right),\left(f_{2}-g_{2}\right)\right\rangle \\
& \quad=-i\left(\left\langle f_{1}, f_{2}\right\rangle-\left\langle f_{1}, g_{2}\right\rangle+\left\langle g_{1}, f_{2}\right\rangle-\left\langle g_{1}, g_{2}\right\rangle\right) .
\end{aligned}
$$

Since $\mathscr{V}$ is an isometry, for any $\left(f_{1}, g_{1}\right),\left(f_{2}, g_{2}\right) \in$ $\mathscr{V},\left\langle f_{1}, f_{2}\right\rangle=\left\langle g_{1}, g_{2}\right\rangle$, this implies that

$$
\begin{aligned}
\left\langlei \left( f_{1}\right.\right. & \left.\left.+g_{1}\right),\left(f_{2}-g_{2}\right)\right\rangle \\
& =-i\left\langle g_{1}, g_{2}\right\rangle+i\left\langle f_{1}, g_{2}\right\rangle-i\left\langle g_{1}, f_{2}\right\rangle+i\left\langle f_{1}, f_{2}\right\rangle \\
& =-i\left(\left\langle g_{1}-f_{1}, g_{2}\right\rangle+\left\langle g_{1}-f_{1}, f_{2}\right\rangle\right) \\
& =\left\langle f_{1}-g_{1}, i\left(f_{2}+g_{2}\right)\right\rangle .
\end{aligned}
$$

Hence, $\mathscr{R}$ is symmetric.

Theorem 4. A symmetric relation $\mathscr{R}$ is self-adjoint if and only if $\mathscr{V}$ is unitary.

Proof. We show that the $\mathscr{R}$ is self-adjoint if and only if

$$
R(\mathscr{R}+i)=R(\mathscr{R}-i)=\mathscr{H} .
$$

Since $\mathscr{R}$ is symmetric, we always have $\mathscr{R} \subset \mathscr{R}^{*}$. Let $(f, g) \in$ $\mathscr{R}^{*}$; then if $-g \in \mathscr{H}$ and $R(\mathscr{R}-i)=\mathscr{H}$ imply that there is $(h, k) \in \mathscr{R}$ such that $k-i h=i f-g$. So $i(f+h)=k+g$, so that $(f+h, i(f+h)) \in \mathscr{R}^{*}$. That is

$$
(f+h) \in N\left(\mathscr{R}^{*}, i\right)=R\left((\mathscr{R}+i)^{\perp}\right)=\{0\} .
$$

This implies $f=-h \in D(\mathscr{R})$. Hence, $\mathscr{R}$ is self-adjoint.

Conversely, suppose that $\mathscr{R}$ is self-adjoint. Let

$$
h \in R(\mathscr{R}-i)^{\perp}=N\left(\mathscr{R}^{*},-i\right)=N(\mathscr{R},-i) .
$$

So $(h,-i h) \in \mathscr{R}$. But

$$
\langle-i h, h\rangle=\langle h, i h\rangle \Longrightarrow i\langle h, h\rangle=-i\langle h, h\rangle .
$$

Hence, we must have $h=0$. So $R(\mathscr{R}-i)=\mathscr{H}$. Similarly, $R(\mathscr{R}+i)=\mathscr{H}$.

Theorem 5. Let $\mathscr{R}$ be a closed symmetric relation on a Hilbert space $\mathscr{H}$ and let $\mathscr{V}$ denote its Cayley transform. One has the following.

(1) $\mathscr{V}^{\prime}$ is the Cayley transform of a closed symmetric extension $\mathscr{R}^{\prime}$ of $\mathscr{R}$ if and only if the following holds. There exist closed subspaces $F_{-}$of $R(\mathscr{R}-i)^{\perp}$ and $F_{+}$of $R(\mathscr{R}+i)^{\perp}$ and an isometric relation $\widetilde{\mathscr{V}}$ on $F_{+} \oplus F_{-}$for which

$$
\begin{gathered}
\mathscr{V}^{\prime}=\{(f+h, g+k):(f, g) \in \mathscr{V},(h, k) \in \widetilde{\mathscr{V}}\}, \\
D\left(\mathscr{V}^{\prime}\right)=R\left(\mathscr{R}^{\prime}+i\right)=R(\mathscr{R}+i) \oplus F_{+}, \\
R\left(\mathscr{V}^{\prime}\right)=R\left(\mathscr{R}^{\prime}-i\right)=R(\mathscr{R}-i) \oplus F_{-} .
\end{gathered}
$$

The spaces $F_{+}$and $F_{-}$have the same dimension.
(2) The relation $\mathscr{V}^{\prime}$ in part (1) is unitary if and only if $F_{-}=$ $R(\mathscr{R}-i)^{\perp}$ and $F_{+}=R(\mathscr{R}+i)^{\perp}$.

(3) $\mathscr{R}$ possess self-adjoint extension if and only if its defect indices are equal.

Proof. (1) Suppose that $\mathscr{V}^{\prime}$ has the given form. Then $\mathscr{V}^{\prime}$ is isometric relation, since for any $(f+h, g+k) \in \mathscr{V}^{\prime}$, we have

$$
\|g+k\|^{2}=\|g\|^{2}+\|k\|^{2}=\|f\|^{2}+\|h\|^{2}=\|f+h\|^{2} .
$$

Hence, we can define a symmetric extension $\mathscr{R}^{\prime}$ such that $\mathscr{V}^{\prime}$ is its Cayley transform. Conversely, if $\mathscr{V}^{\prime}$ is the Cayley transform of a symmetric extension $\mathscr{R}^{\prime}$ of $\mathscr{R}$, then put $F_{-}=$ $R\left(\mathscr{R}^{\prime}-i\right) \ominus R(\mathscr{R}-i), F_{+}=R\left(\mathscr{R}^{\prime}+i\right) \ominus R(\mathscr{R}+i)$ and $\widetilde{\mathscr{V}}=\left.\mathscr{V}^{\prime}\right|_{F_{+} \oplus F_{-}}$. Then we have the desired properties.

(2) Here we have that $\mathscr{V}^{\prime}$ is unitary if and only if

$$
D\left(\mathscr{V}^{\prime}\right)=R\left(\mathscr{V}^{\prime}\right)=\mathscr{H} .
$$

That is, if and only if $F_{-}=R(\mathscr{R}-i)^{\perp}$ and $F_{+}=R(\mathscr{R}+i)^{\perp}$.

(3) By (1) and (2), $\mathscr{V}$ possess unitary extension if and only if there exists an isometry relation $\widetilde{\mathscr{V}}$ onto $R(\mathscr{R}+i)^{\perp} \oplus R(\mathscr{R}-$ $i)^{\perp}$. This happens if and only if

$$
\operatorname{dim}\left(R(\mathscr{R}+i)^{\perp}\right)=\operatorname{dim}\left(R(\mathscr{R}-i)^{\perp}\right) .
$$

By definition of Cayley transform, it is clear that if $\mathscr{V}_{1}$ and $\mathscr{V}_{2}$ are the Cayley transforms of any two symmetric relations $\mathscr{R}_{1}$ and $\mathscr{R}_{2}$, then

$$
\mathscr{R}_{1} \subset \mathscr{R}_{2} \text { if and only if } \mathscr{V}_{1} \subset \mathscr{V}_{2} .
$$

Theorem 6. Let $\mathscr{R}$ be a closed symmetric relation on a Hilbert space with defect indices $(m, m)$. One has the following.

(1) $\mathscr{R}^{\prime}$ is a symmetric extension of $\mathscr{R}$ if and only if the following holds. There are closed subspaces $F_{+}$of $R(i+$ $\mathscr{R})^{\perp}$ and $F_{-}$of $R(i-\mathscr{R})^{\perp}$ and an isometric mapping $\widehat{\mathscr{V}}$ of $F_{+}$onto $F_{-}$such that

$$
D\left(\mathscr{R}^{\prime}\right)=D(\mathscr{R})+\left\{g+\widehat{\mathscr{V}} g: g \in F_{+}\right\} .
$$

(2) $\mathscr{R}^{\prime}$ is self-adjoint if and only if $\mathscr{R}^{\prime}$ is an $m$-dimensional extension of $\mathscr{R}$.

Proof. (1) Let $\mathscr{V}$ and $\mathscr{V}^{\prime}$ be the Cayley transforms of the closed symmetric relation $\mathscr{R}$ and its symmetric extension $\mathscr{R}^{\prime}$, respectively. By Theorem 5 , there exist closed subspaces $F_{-}$of $R(\mathscr{R}-i)^{\perp}$ and $F_{+}$of $R(\mathscr{R}+i)^{\perp}$ and an isometric relation $\widetilde{\mathscr{V}}$ on $F_{+} \oplus F_{-}$for which

$$
\begin{gathered}
\mathscr{V}^{\prime}=\{(f+h, g+k):(f, g) \in \mathscr{V},(h, k) \in \widetilde{\mathscr{V}}\}, \\
D\left(\mathscr{V}^{\prime}\right)=R\left(\mathscr{R}^{\prime}+i\right)=R(\mathscr{R}+i) \oplus F_{+}, \\
R\left(\mathscr{V}^{\prime}\right)=R\left(\mathscr{R}^{\prime}-i\right)=R(\mathscr{R}-i) \oplus F_{-} .
\end{gathered}
$$


Then by definition of the Cayley transform, we see that

$$
\begin{aligned}
D\left(\mathscr{R}^{\prime}\right) & =R\left(I-\mathscr{V}^{\prime}\right)=\left(I-\mathscr{V}^{\prime}\right) D\left(\mathscr{V}^{\prime}\right) \\
& =\left(I-\mathscr{V}^{\prime}\right) R\left(i+\mathscr{R}^{\prime}\right) \\
& =\left(I-\mathscr{V}^{\prime}\right)\left(R(i+\mathscr{R}) \oplus F_{+}\right) \\
& =\left(I-\mathscr{V}^{\prime}\right)\left(D(\mathscr{V}) \oplus F_{+}\right) \\
& =(I-\mathscr{V}) D(\mathscr{V})+\left(I-\mathscr{V}^{\prime}\right) F_{+} \\
& =D(\mathscr{R})+\left\{g-\widetilde{\mathscr{V}} g: g \in F_{+}\right\} .
\end{aligned}
$$

The converse is similar.

(2) By Theorem $5, \mathscr{R}^{\prime}$ is self-adjoint if and only if $\mathscr{V}^{\prime}$ is unitary. This happens if and only if $F_{+}=R(\mathscr{R}+i)^{\perp}$. So, by (1), $\mathscr{R}^{\prime}$ is self-adjoint if and only if it is an $m$-dimensional extension of $\mathscr{R}$.

Theorem 7. Suppose that $\mathscr{T}$ is a self-adjoint relation and suppose that $z \in \Gamma(\mathscr{T})$; then

$$
\mathscr{H}=\{z f-g:(f, g) \in \mathscr{T}\} .
$$

Proof. We will show that $R(z-\mathscr{T})=\{z f-g:(f, g) \in \mathscr{T}\}$ is a closed subspace of $\mathscr{H}$. Since $z \in \Gamma(\mathscr{T})$, there is a constant $C(z)$ such that

$$
\|z f-g\| \geq C(z)\|f\|
$$

Let $v_{n} \in R(z-\mathscr{T})$ and $v_{n} \rightarrow v$ in $\mathscr{H}$. Suppose that $f_{n} \in D(\mathscr{T})$ such that $\left(f_{n}, g_{n}\right) \in \mathscr{T}$ and $v_{n}=z f_{n}-g_{n}$ so that $\left(f_{n}, v_{n}\right) \in$ $z-\mathscr{T}$. But from the above relation we have

$$
\left\|v_{n}-v_{m}\right\|=\left\|z\left(f_{n}-f_{m}\right)-\left(g_{n}-g_{m}\right)\right\| \geq C\left\|f_{n}-f_{m}\right\| .
$$

It follows that $f_{n}$ is a Cauchy sequence in $\mathscr{H}$, and it converges to some $f$ in $\mathscr{H}$. Hence, $\left(f_{n}, v_{n}\right) \rightarrow(f, v)$. Since $\mathscr{T}$ is closed, $f \in D(\mathscr{T})$ and $(f, v) \in z-\mathscr{T}$ and $v \in R(z-\mathscr{T})$. Hence, $R(z-\mathscr{T})$ is closed. So we have

$$
\mathscr{H}=R(z-\mathscr{T}) \oplus R(z-\mathscr{T})^{\perp} .
$$

We next show that $R(z-\mathscr{T})^{\perp}=\{0\}$. Let $h \in R(z-\mathscr{T})^{\perp}=$ $N(\mathscr{T}, \bar{z})$; then $(h, \bar{z} h) \in \mathscr{T}$. But $0=\|\bar{z} h-\bar{z} h\| \geq C(\bar{z})\|h\|$ implies $h=0$ a. e..

Let $\mathscr{T}$ be a self-adjoint relation on $\mathscr{H}$ and $z \in \Gamma(\mathscr{T})$. Define $T: \mathscr{H} \rightarrow \mathscr{H}$ by $T(z f-g)=f$. That is $T=(z-\mathscr{T})^{-1}=$ $\{(z f-g, f):(f, g) \in \mathscr{T}\}$. Then $T$ is a bounded linear operator since

$$
\|T\|=\sup _{\|z f-g\|=1}\|T(z f-g)\|=\sup _{\|z f-g\|=1}\|f\| \leq \frac{1}{C(z)}
$$

and $\mathscr{T}$ is given by

$$
\mathscr{T}=\{(T f, z T f-f): f \in \mathscr{H}\} .
$$

\section{Spectral Theory of a Linear Relation}

Definition 8. Let $\mathscr{R}$ be a closed relation on a Hilbert space $\mathscr{H}$. We define

$$
\begin{aligned}
\rho(\mathscr{R})=\{z \in \mathbb{C}: & \exists T \in B(\mathscr{H}), \\
\mathscr{R} & =\{(T f, z T f-f): f \in \mathscr{H}\}\}
\end{aligned}
$$

to be the resolvent set and $\sigma(\mathscr{R})=\mathbb{C}-\rho(\mathscr{R})$ to be the spectrum of $\mathscr{R}$.

Remark 9. When a relation $\mathscr{R}$ is an operator on $\mathscr{H}$, then

$$
\rho(\mathscr{R})=\left\{z \in \mathbb{C}:(z-\mathscr{R})^{-1} \in B(\mathscr{H})\right\} .
$$

A complex number $z \in \mathbb{C}$ is called an eigenvalue of a relation $\mathscr{R}$ if there exists $f \in \mathscr{H}, f \neq 0$ such that $(f, z f) \in \mathscr{R}$. The set of all eigenvalues of $\mathscr{R}$ is called the point spectrum of $\mathscr{R}$ and is denoted by $\sigma_{p}(\mathscr{R})$.

Remark 10. For any closed relation $\mathscr{R}$ on a Hilbert space $\mathscr{H}$, $\sigma_{p}(\mathscr{R}) \subset \sigma(\mathscr{R})$.

Let $\mathscr{Z}=\{(0, g) \in \mathscr{R}\}$ and $Z=\{g:(0, g) \in$ $\mathscr{R}\}$ be the multivalued part of a relation $\mathscr{R}$. Clearly $Z$ is a closed subspace of $\mathscr{H}$. Note that $D(\mathscr{R})$ is not dense if $\mathscr{R}$ is multivalued. Now define the quotient space $\mathscr{H}_{s}=\mathscr{H} / Z$. We know that this quotient space is also a Hilbert space with the norm defined by

$$
\|[f]\|=\inf _{g \in Z}\|f+g\| .
$$

Define a relation $\mathscr{R}_{s}$ on $\mathscr{H}_{s} \oplus \mathscr{H}_{s}$ by $\mathscr{R}_{s}=\{([f],[g]):(f, g) \epsilon$ $\mathscr{R}\}$. We consider the relation $\mathscr{R}_{s}$ as the restriction of $\mathscr{R}$ on $\mathscr{H}_{s}$. By natural isomorphism, the space $\mathscr{H}_{s}$ is identified as $\mathscr{H} \ominus Z$ and the relation $\mathscr{R}_{s}$ as $\mathscr{R} \ominus \mathscr{Z}$. Then clearly $\mathscr{R}_{s}$ is an operator on $\mathscr{H}_{s}$ with $D\left(\mathscr{R}_{s}\right)=D(\mathscr{R})$.

Theorem 11. If $\mathscr{T}$ is a self-adjoint relation on $\mathscr{H}$, then

$$
S(\mathscr{T})=\sigma(\mathscr{T})=\sigma\left(\mathscr{T}_{s}\right) .
$$

Proof. Letting $z \in \Gamma(\mathscr{T})$, then there exists a constant $C>0$ such that

$$
\|z f-g\| \geq C\|f\|, \quad \forall(f, g) \in \mathscr{T} .
$$

For such $z$, we can define $T=(z-\mathscr{T})^{-1}$ as a bounded linear operator on $\mathscr{H}$ such that $\mathscr{T}=\{(T h, z T h-h): h \in \mathscr{H}\}$. So $z \in \rho(\mathscr{T})$. On the other hand, let $z \in \rho(\mathscr{T})$; then there exists $T \in B(\mathscr{H})$ such that $\mathscr{T}=\{(T h, z T h-h): h \in \mathscr{H}\}$. For any $(f, g) \in \mathscr{T}$, there is $h \in \mathscr{H}$ such that $T h=f$ and $z T h-h=g$. So

$$
\|z f-g\|=\|z T h-z T h+h\|=\|h\| \geq C\|T h\|=C\|f\|
$$

for some $C>0$ and hence $z \in \Gamma(\mathscr{T})$. Hence, $S(\mathscr{T})=\sigma(\mathscr{T})$. Next, assuming that $z \in \Gamma\left(\mathscr{T}_{s}\right)$, then for any $([f],[g]) \in \mathscr{T}_{s}$, there exists a constant $C>0$ such that

$$
\|z[f]-[g]\| \geq C\|[f]\| .
$$


For any $(f, g) \in \mathscr{T}$ we have

$$
\|z f-g\| \geq\|z[f]-[g]\| \geq C\|[f]\|=C\|f\| .
$$

Hence $z \in \Gamma(\mathscr{T})$. On the other hand, suppose that $z \in \Gamma(\mathscr{T})$, then there is a constant $C>0$ such that

$$
\|z f-g\| \geq C\|f\| \text {. }
$$

For any $([f],[g]) \in \mathscr{T}_{s}$, we have

$$
\begin{aligned}
\|z[f]-[g]\| & =\inf _{u \in Z}\|z f-g+u\| \\
& =\inf _{u \in Z}(\|z f-g\|+\|u\|) \\
& \geq\|z f-g\| \geq C\|f\|=C\|[f]\| .
\end{aligned}
$$

This implies that $z \in \Gamma\left(\mathscr{T}_{s}\right)$. Thus, $S\left(\mathscr{T}_{s}\right)=S(\mathscr{T})$. Hence, $S(\mathscr{T})=\sigma(\mathscr{T})=\sigma\left(\mathscr{T}_{s}\right)$.

Remark 12. If $\mathscr{T}$ is a self-adjoint relation on $\mathscr{H}$, then $\sigma(\mathscr{T}) \subset$ $\mathbb{R}$.

Theorem 13. Let $z \in \Gamma(\mathscr{T})$ and $T=(z-\mathscr{T})^{-1}$. One has the following.

(1) If $\lambda \in \Gamma(T)$, then $(z-(1 / \lambda)) \in \Gamma(\mathscr{T})$.

(2) If $\lambda \in S(\mathscr{T})$, then $(1 /(z-\lambda)) \in S(T)$.

(3) $S(T) \subset \sigma(T)$.

Proof. (1) Let $\lambda \in \Gamma(T)$; then by definition there exists $C(\lambda)>$ 0 such that

$$
\|\lambda(z f-g)-f\| \geq C(\lambda)\|z f-g\|, \quad \forall(f, g) \in \mathscr{T} .
$$

Note that $\lambda \neq 0$. For any $(f, g) \in \mathscr{T}$, we have

$$
\begin{aligned}
\left\|\left(z-\frac{1}{\lambda}\right) f-g\right\| & =\frac{1}{|\lambda|}\|z \lambda f-f-\lambda g\| \\
& =\frac{1}{|\lambda|}\|\lambda(z f-g)-f\| \\
& \geq \frac{C(\lambda)}{|\lambda|}\|z f-g\| \geq \frac{C(\lambda) C(z)}{|\lambda|}\|f\| .
\end{aligned}
$$

So $(z-(1 / \lambda)) \in \Gamma(\mathscr{T})$.

(2) Let $\lambda \in S(\mathscr{T})$ and suppose that $(1 /(z-\lambda)) \notin S(T)$. Then $(1 /(z-\lambda)) \in \Gamma(T)$. But by $(1),(z-1 /(1 /(z-\lambda))) \in \Gamma(\mathscr{T})$. This implies that $\lambda \in \Gamma(\mathscr{T})$ which is a contradiction.

(3) Let $\lambda \in \rho(T)$; then $(\lambda-T)^{-1}$ is bounded and is defined on all of $\mathscr{H}$; then for any $(f, g) \in \mathscr{T}$

$$
\begin{aligned}
\|z f-g\| & =\left\|(\lambda-T)^{-1}(\lambda-T)(z f-g)\right\| \\
& \leq\left\|(\lambda-T)^{-1}\right\|\|\lambda(z f-g)-T(z f-g)\| \\
& \Longrightarrow\|\lambda(z f-g)-T(z f-g)\| \\
& \geq \frac{1}{\left\|(\lambda-T)^{-1}\right\|}\|z f-g\| .
\end{aligned}
$$

Note. We may think of developing further the spectral theory of linear relation analogous to that of operator theory. Any extension would be useful in many of the application problems which induce linear relation instead of linear operator.

\section{Conflict of Interests}

The author declares that there is no conflict of interests regarding the publication of this paper.

\section{References}

[1] R. Arens, "Operational calculus of linear relations," Pacific Journal of Mathematics, vol. 11, pp. 9-23, 1961.

[2] E. A. Coddington, Extension Theory of Formally Normal and Symmetric Subspaces, Memoirs of the American Mathematical Society, no. 134, American Mathematical Society, Providence, RI, USA, 1973.

[3] A. Dijksma and H. S. V. de Snoo, "Self-adjoint extensions of symmetric subspaces," Pacific Journal of Mathematics, vol. 54, pp. 71-100, 1974.

[4] H. Langer and B. Textorius, "On generalized resolvents and Qfunctions of symmetric linear relations (subspaces) in Hilbert space," Pacific Journal of Mathematics, vol. 72, no. 1, pp. 135-165, 1977.

[5] A. Favini and A. Yagi, Degenerate Differential Equations in Banach Spaces, vol. 215 of Monographs and Textbooks in Pure and Applied Mathematics, Marcel Dekker, New York, NY, USA, 1999.

[6] S. Hassi, H. de Snoo, and H. Winkler, "Boundary-value problems for two-dimensional canonical systems," Integral Equations and Operator Theory, vol. 36, no. 4, pp. 445-479, 2000.

[7] S. Hassi, C. Remling, and H. de Snoo, "Subordinate solutions and spectral measures of canonical systems," Integral Equations and Operator Theory, vol. 37, no. 1, pp. 48-63, 2000.

[8] J. Weidmann, Linear Operators in Hilbert Spaces, vol. 68 of Graduate Texts in Mathematics, Springer, New York, NY, USA, 1980.

[9] C. Remling, "Schrödinger operators and de Branges spaces," Journal of Functional Analysis, vol. 196, no. 2, pp. 323-394, 2002.

$\Rightarrow \lambda \in \Gamma(T)$. This shows that $S(T) \subset \sigma(T)$. 


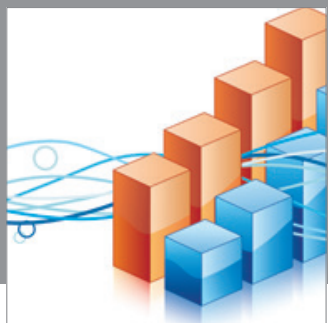

Advances in

Operations Research

mansans

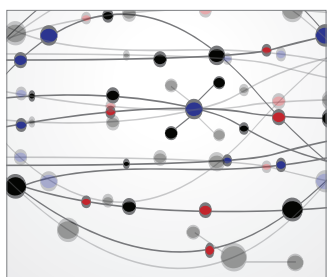

The Scientific World Journal
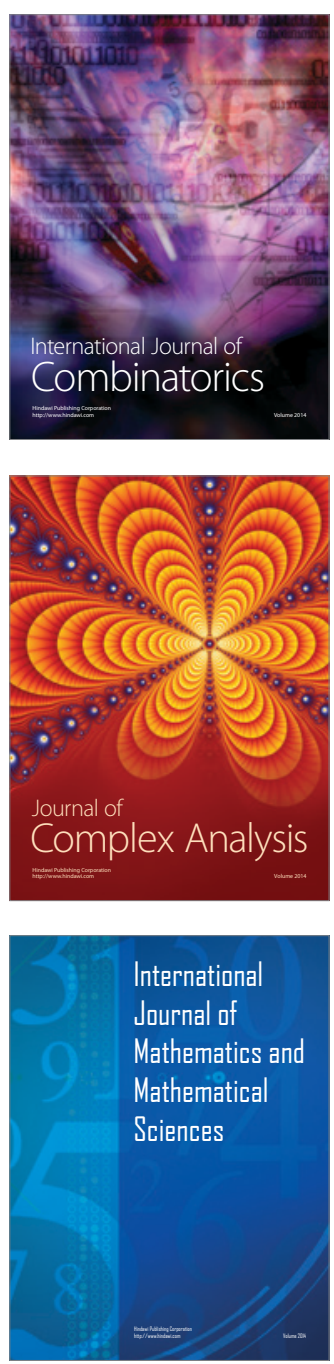
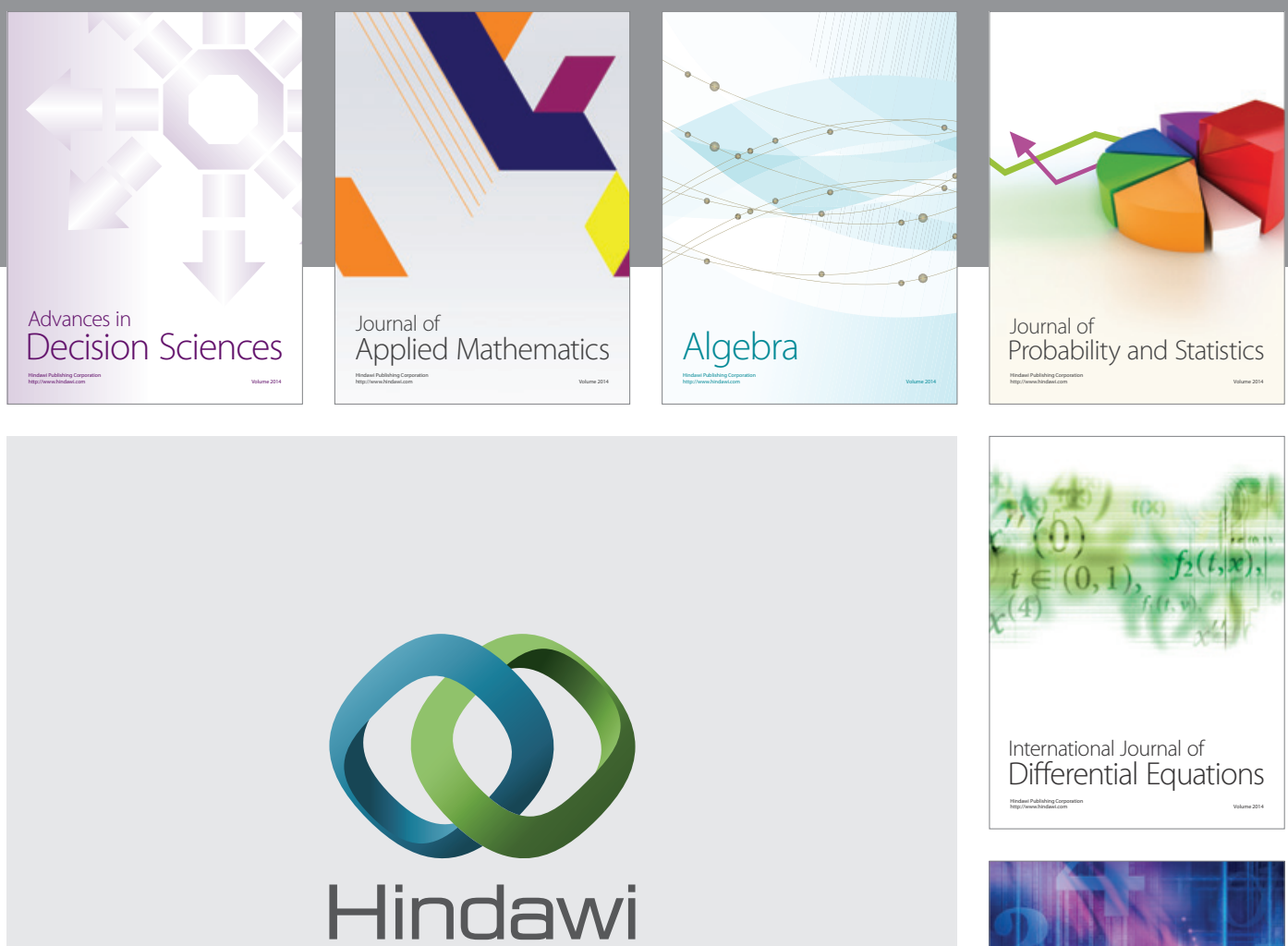

Submit your manuscripts at http://www.hindawi.com
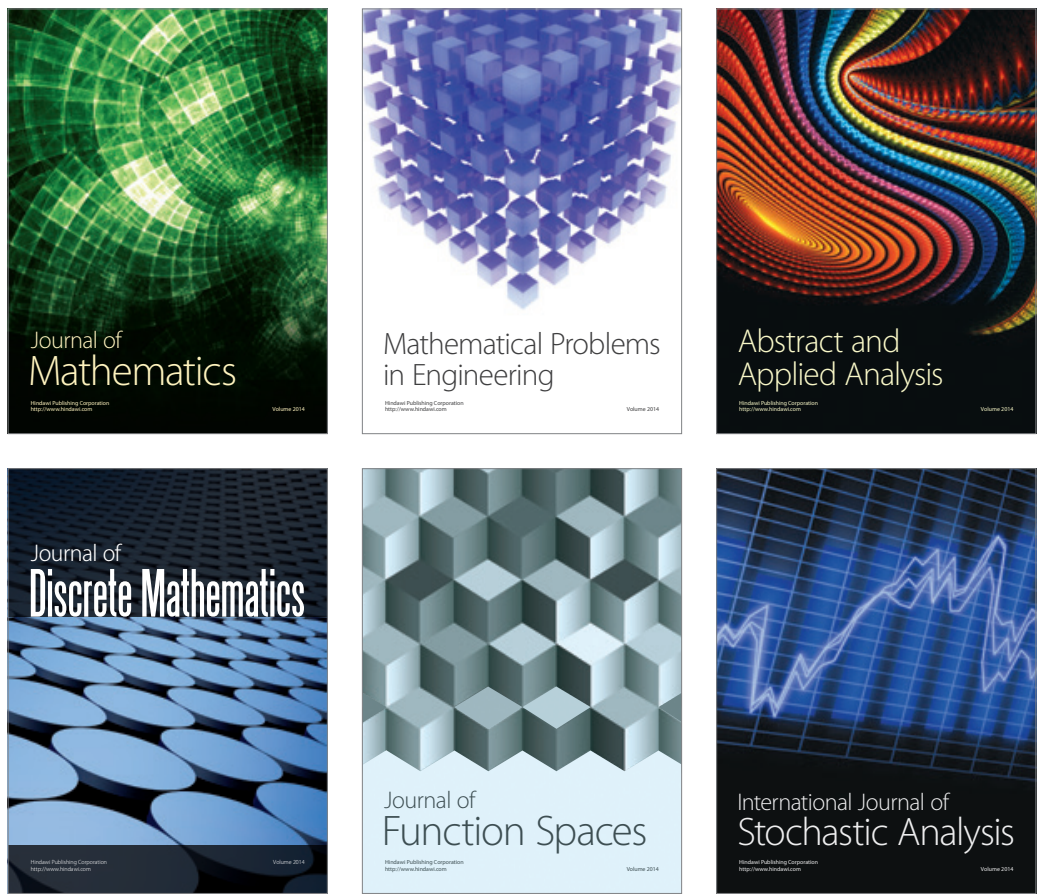

Journal of

Function Spaces

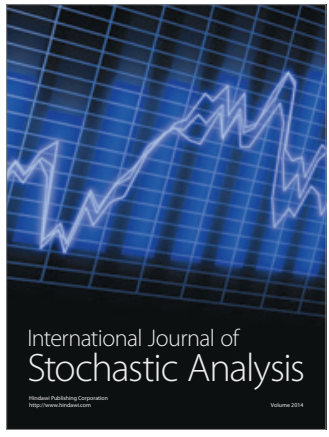

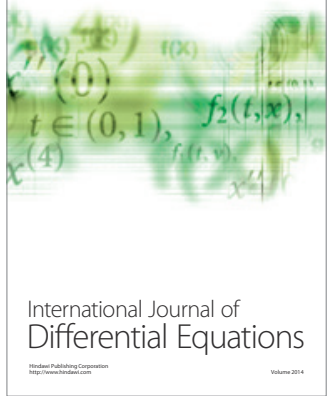
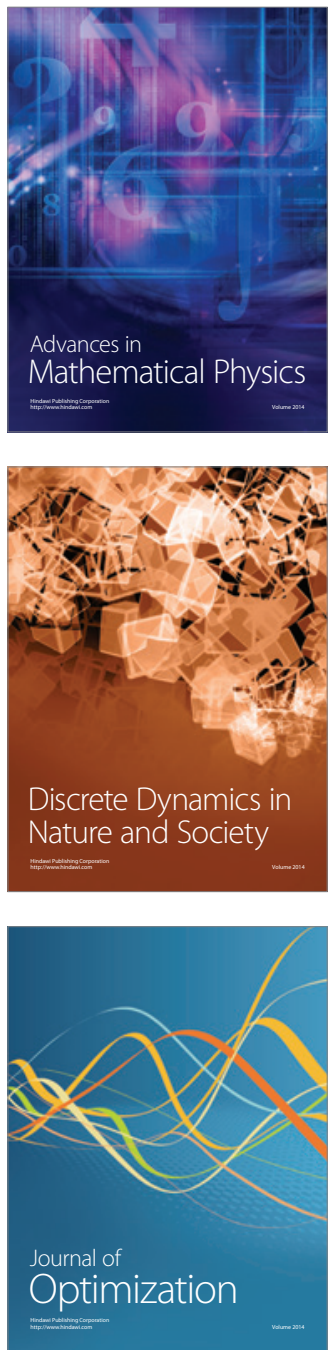